\title{
Thermodynamics of AdS Black Holes: Critical Behavior of the Central Charge
}

\author{
Wan Cong, ${ }^{1,2, *}$ David Kubizňák $\odot,{ }^{2,1, \dagger}$ and Robert B. Mann ${ }^{1,2, \$}$ \\ ${ }^{1}$ Department of Physics and Astronomy, University of Waterloo, Waterloo, Ontario N2L 3G1, Canada \\ ${ }^{2}$ Perimeter Institute, 31 Caroline Street North, Waterloo, Ontario N2L 2Y5, Canada
}

(Received 10 June 2021; accepted 28 July 2021; published 23 August 2021)

\begin{abstract}
We reconsider the thermodynamics of anti-de Sitter black holes in the context of gauge-gravity duality. In this new setting, where both the cosmological constant $\Lambda$ and the gravitational Newton's constant $G$ are varied in the bulk, we rewrite the first law in a new form containing both $\Lambda$ (associated with thermodynamic pressure) and the central charge $C$ of the dual conformal field theory and their conjugate variables. We obtain a novel thermodynamic volume, in turn leading to a new understanding of the Van der Waals behavior of charged anti-de Sitter black holes in which phase changes are governed by the degrees of freedom in the conformal field theory. Compared to the "old" $P-V$ criticality, this new criticality is "universal" (independent of the bulk pressure) and directly relates to the thermodynamics of the dual field theory and its central charge.
\end{abstract}

DOI: 10.1103/PhysRevLett.127.091301

Black holes and their thermodynamics have been of crucial importance in providing clues about the nature of quantum gravity. Of particular interest are asymptotically anti-de Sitter (AdS) black holes, which provide a description of the dual conformal field theory (CFT) at finite temperature via the AdS/CFT correspondence [1]. Such black holes can be in thermal equilibrium with their Hawking radiation and exhibit interesting thermodynamic phase transitions such as the first order Hawking-Page phase transition [2], which corresponds to the confinement or deconfinement of the dual quark gluon plasma [3], or the existence of a second order Van der Waals type phase transition for charged AdS black holes [4-7].

One of the more interesting developments in recent years has been a realization that a negative cosmological constant $\Lambda$ induces a positive thermodynamic pressure $P$ :

$$
P=-\frac{\Lambda}{8 \pi G}, \quad \Lambda=-\frac{(D-1)(D-2)}{2 l^{2}},
$$

upon which the thermodynamics of AdS black holes becomes more complete, i.e., "identical" to the thermodynamics of ordinary systems (including the $P \delta V$ term) [7]. Here, $l$ is the radius of the $D$-dimensional AdS space and $G$ is the (dimensionful) gravitational Newton constant; we set $\hbar=c=1$.

Published by the American Physical Society under the terms of the Creative Commons Attribution 4.0 International license. Further distribution of this work must maintain attribution to the author(s) and the published article's title, journal citation, and DOI. Funded by SCOAP ${ }^{3}$.
In this "extended thermodynamic phase space" [8], the black hole mass $M$ is interpreted as thermodynamic enthalpy rather than internal energy, and the first law of thermodynamics and the corresponding Smarr relation for a black hole of charge $Q$, surface gravity $\kappa$, angular momentum $J$, and area $A$ are

$$
\begin{gathered}
\delta M=T \delta S+V \delta P+\phi \delta Q+\Omega \delta J \\
M=\frac{D-2}{D-3}(T S+\Omega J)+\phi Q-\frac{2}{D-3} P V,
\end{gathered}
$$

where entropy $S$ and temperature $T$ are

$$
S=\frac{A}{4 G}, \quad T=\frac{\kappa}{2 \pi},
$$

with $\phi, \Omega$ the respective conjugates to $Q, J$, and where

$$
V=\left(\frac{\partial M}{\partial P}\right)_{S, Q, J}
$$

is the thermodynamic volume conjugate to $P[9,10]$.

In this framework, black hole thermodynamics is phenomenologically much richer than previously expected, with black holes exhibiting Van der Waals [7], reentrant [11], superfluid [12], and polymer-type phase transitions [13], along with triple points $[14,15]$ and the universal scaling behavior of the Ruppeiner curvature [16]. For these reasons, this subdiscipline has come to be called black hole chemistry [17].

The "holographic interpretation" of black hole chemistry has been somewhat elusive [18-24]. The first law, Eq. (2), cannot be straightforwardly related to the corresponding 
thermodynamics of the holographic dual field theory [25-27] because variations of the bulk cosmological constant $\Lambda$ correspond to changing both the central charge $C$ (or the number of colors $N$ ) and the CFT volume $\mathcal{V}$. Indeed, it also corresponds to changing the notion of electric charge and the corresponding chemical potential that both rescale with the AdS radius $l$. It is possible to hold $C$ fixed so that the field theory remains the same (in which case varying $\Lambda$ has the more natural consequence of varying $\mathcal{V}$ of the CFT) by simultaneously varying Newton's constant $G$. (Alternative reasons as to why $G$ should be varied in the first law of black hole thermodynamics have already been put forward [28].)

Here, we demonstrate that variation of $G$ has profound consequences for black hole chemistry and its holographic interpretation. We build on previous holographic generalizations [27] of the first law, Eq. (2), which include variations of $G$ by rewriting it in a new "mixed" form [29], in terms of variations of the bulk pressure $P$ and the CFT central charge $C$. The explicit appearance of $\delta C$ allows for a study of bulk thermodynamics in the same CFT theory on the AdS boundary and yields a new definition for the thermodynamic black hole volume. Surprisingly, as we shall see, this also leads to a novel understanding of phase transitions of (charged) AdS black holes that, contrary to the conclusions of black hole chemistry, no longer necessarily depends on the bulk critical pressure but rather derives from the critical central charge of the dual CFT.

We begin by considering the correspondence between the holographic CFT and bulk thermodynamic quantities valid in Einstein gravity (large $N$ limit) $[25,27]$. For the CFT, the first law is [27]

$$
\delta E=T \delta S-p d \mathcal{V}+\tilde{\phi} \delta \tilde{Q}+\Omega \delta J+\tilde{\mu} \delta C,
$$

where $E$ is the CFT energy (not enthalpy), $p$ and $\mathcal{V}=\mathcal{V}_{0} l^{D-2}$ are the CFT pressure and volume, $\tilde{\mu}$ is the chemical potential for the central charge $C$, which is proportional to $N$ to some power $\left[C \propto N^{2}\right.$ for $S U(N)$ gauge theories with conformal symmetry], $J$ and $\Omega$ are the angular momentum and conjugate angular velocity, and $\tilde{Q}$, $\tilde{\phi}$ are its holographic charge and conjugate potential, respectively. These quantities scale as

$$
\begin{aligned}
& {[E]=[T]=[\Omega]=[\tilde{\mu}]=\frac{1}{L}, \quad[\mathcal{V}]=L^{D-2},} \\
& {[S]=[\tilde{Q}]=[J]=[C]=L^{0},}
\end{aligned}
$$

which in turn implies

$$
E=(D-2) p \mathcal{V}
$$

from the standard dimensional Euler scaling argument.

Using the fact that $E, S, \tilde{Q}, J, C$ scale as $C$ but $\mathcal{V}$ does not [27], a similar Euler argument yields

$$
E=T S+\tilde{\phi} \tilde{Q}+\Omega J+\tilde{\mu} C,
$$

which is the holographic Smarr relation [27]. Note that Eq. (9) has no $D$-dependent factors and that the $p-\mathcal{V}$ term does not appear here.

In order to obtain a duality between holographic and bulk thermodynamics, we employ the following duality relation:

$$
C=k \frac{l^{D-2}}{16 \pi G}
$$

where the numerical factor $k$ depends on the details of the particular holographic system [25]. We likewise identify

$$
E=M, \quad \tilde{Q}=\frac{Q l}{\sqrt{G}}, \quad \tilde{\phi}=\frac{\phi \sqrt{G}}{l}
$$

and make use of Eq. (1) to identify $\Lambda$ and $l$. Allowing both $l$ and $G$ to vary [27], from Eqs. (6) and (9) we obtain

$$
\begin{aligned}
\delta(G M) & =\frac{\kappa}{8 \pi} \delta A+\Omega \delta(G J)+\sqrt{G} \phi \delta(\sqrt{G} Q)-\frac{V}{8 \pi} \delta \Lambda \\
& \Leftrightarrow \delta M=\frac{\kappa}{8 \pi G} \delta A+\Omega \delta J+\phi \delta Q-\frac{V}{8 \pi G} \delta \Lambda-\alpha \frac{\delta G}{G},
\end{aligned}
$$

where

$$
\begin{aligned}
& \alpha=\frac{1}{2} \phi Q+\tilde{\mu} C+T S=M-\Omega J-\frac{1}{2} \phi Q, \\
V= & \frac{8 \pi G l^{2}}{(D-1)(D-2)}[M-\phi Q-(D-2) C \tilde{\mu}] \\
= & \frac{(D-3)}{2 P}\left[\frac{D-2}{D-3}(\Omega J+T S)+\phi Q-M\right] .
\end{aligned}
$$

We see that Eq. (14) is equivalent to the bulk Smarr relation, Eq. (3), and when $G$ is held fixed the first law, Eq. (12), reduces to Eq. (2).

The quantity $\alpha / G$ is a thermodynamic conjugate to $G$, and the factor in front of the $\phi Q$ term in Eq. (13) differs from that presented previously [27] due to the $G$ dependence of $\tilde{Q}$ and $\tilde{\phi}$ of the dual CFT, cf. Eq. (11). This difference can be attributed to a different " $G$ normalization" of the action: in [27], the "AdS/CFT conventions" [4] were followed; here, we employ standard "gravitational physics normalization" [30], in which the stress-energy contribution to the Einstein equations vanishes in the limit $G \rightarrow 0$.

Since $G$ is now a thermodynamic variable, dimensional analysis implies 


$$
\begin{array}{ll}
{[\phi]=L^{\frac{2-D}{2}},} & {[Q]=L^{\frac{D-4}{2}}, \quad[A]=[G]=L^{D-2},} \\
{[M]=\frac{1}{L},} & {[\Lambda]=\frac{1}{L^{2}}, \quad[P]=\frac{1}{L^{D}}, \quad[V]=L^{D-1},}
\end{array}
$$

from which one can recover the bulk Smarr relation, Eq. (3), provided $\alpha$ is given as in Eq. (13). Notice that this way of recovering Eq. (3) required input from the CFT side since Eq. (13) follows from the holographic Smarr relation. An alternative way to obtain this without reference to the CFT would be to make use of the dependence

$$
G M=\mathcal{M}(A, \sqrt{G} Q, G J, \Lambda)
$$

in the bulk. Its differential, together with the first law, Eq. (12), then yields the expression for $\alpha$, Eq. (13), which in turn yields Eq. (3) by the usual dimensional scaling argument.

We would now rewrite Eq. (12) in terms of variations in the bulk pressure $P$. We can do so by making use of

$$
\frac{\delta G}{G}=-\frac{2}{D} \frac{\delta C}{C}-\frac{D-2}{D} \frac{\delta P}{P},
$$

which follows from Eq. (10). Substituting this into Eq. (12) allows us to write the bulk first law in the following "mixed" form:

$$
\delta M=T \delta S+\Omega \delta J+\phi \delta Q+V_{C} \delta P+\mu \delta C,
$$

where

$$
V_{C}=\frac{2 M+(D-4) \phi Q}{2 D P}, \quad \mu=\frac{2 P\left(V_{C}-V\right)}{C(D-2)}
$$

are the new thermodynamic volume $V_{C}$ and chemical potential $\mu$. This new form of the first law allows us to study the bulk thermodynamics with the familiar pressurevolume term from traditional black hole chemistry, while at the same time keeping the boundary central charge fixed. Note from Eq. (17) that fixing the central charge determines variations of $G$ in terms of those in $P$, whereas fixing $G$ determines variations of $C$ in terms of those in $P$ (while the CFT volume $\mathcal{V}$ changes as well), which then recovers the usual thermodynamics in black hole chemistry [Eq. (2)].

It is well known that for a large class of black holes, the thermodynamic volume $V$ in Eq. (5) satisfies the reverse isoperimetric inequality [10]

$$
\mathcal{R} \equiv\left[\frac{(D-1) V}{\omega_{D-2}}\right]^{\frac{1}{D-1}}\left(\frac{\omega_{D-2}}{A}\right)^{\frac{1}{D-2}} \geq 1,
$$

where $A$ is the horizon area and $\omega_{n}=2 \pi^{[(n+1) / 2]} /$ $\Gamma[(n+1) / 2]$, with the inequality saturated for (simple) spherical black holes. For fixed $G$, this amounts to a statement that Schwarzschild AdS black holes are "maximally entropic" for a given black hole volume $V$. It follows from Eq. (19) (for spherical black holes) that whenever the chemical potential $\mu$ becomes negative, that is, $V>V_{C}$, the standard (rather than reverse) isoperimetric inequality for $V_{C}$ is satisfied. As we shall see below, this happens for large charged AdS black holes-with respect to the volume $V_{C}$, such black holes are therefore "superentropic" [31].

We now test our new notions on a charged AdS black hole, reconsidering its thermodynamic criticality. First studied in the $\phi-Q$ plane [4-6], the analogy between the thermodynamics of charged AdS black holes and Van der Waals fluids was properly completed in the extended phase space [7]. Subsequently, the corresponding CFT criticality in the context of $A d S_{5} \times S_{5}$ was studied $[19,21,22]$ (see also [32]) in the $\tilde{\mu}-C$ plane and also in the $p-\mathcal{V}$ plane [23]. Maxwell's equal area law for the entanglement entropy has likewise been investigated [24].

A charged AdS black hole is characterized by the following metric and gauge potential:

$$
\begin{aligned}
d s^{2} & =-f d t^{2}+\frac{d r^{2}}{f}+r^{2} d \Omega_{2}^{2}, \quad A=-\frac{Q}{r} d t, \\
f & =1-\frac{2 G M}{r}+\frac{G Q^{2}}{r^{2}}+\frac{r^{2}}{l^{2}},
\end{aligned}
$$

from which we find

$$
\begin{aligned}
M & =\frac{r_{+}\left(l^{2}+r_{+}^{2}\right)}{2 l^{2} G}+\frac{Q^{2}}{2 r_{+}}, \quad T=\frac{3 r_{+}^{4}+l^{2} r_{+}^{2}-G Q^{2} l^{2}}{4 \pi l^{2} r_{+}^{3}}, \\
S & =\frac{\pi r_{+}^{2}}{G}, \quad V=\frac{4 \pi r_{+}^{3}}{3}, \quad \phi=\frac{Q}{r_{+}}
\end{aligned}
$$

for the standard thermodynamic quantities, while the new thermodynamic variables are

$$
\begin{aligned}
& V_{C}=\frac{\pi}{3 r_{+}}\left(G Q^{2} l^{2}+l^{2} r_{+}^{2}+r_{+}^{4}\right), \\
& \mu=\frac{2 \pi}{k l^{4} r_{+}}\left(G Q^{2} l^{2}+l^{2} r_{+}^{2}-3 r_{+}^{4}\right) .
\end{aligned}
$$

Small black holes, for which

$$
r_{+}^{2}<\frac{l^{2}}{6}\left(1+\sqrt{1+\frac{12 G Q^{2}}{l^{2}}}\right),
$$

have positive $\mu$ (typical for the quantum regime). Large black holes have large negative $\mu$ (classical regime) and violate the reverse isoperimetric inequality for $V_{C}$ (while this is saturated for $V$ ). We display the corresponding $\mu-T$ diagram (for fixed $P$ and $Q$ ) for various values of $C$ in Fig. 1. The multivaluedness for $C>C_{c}$ indicates the presence of the first order phase transition (see below). 


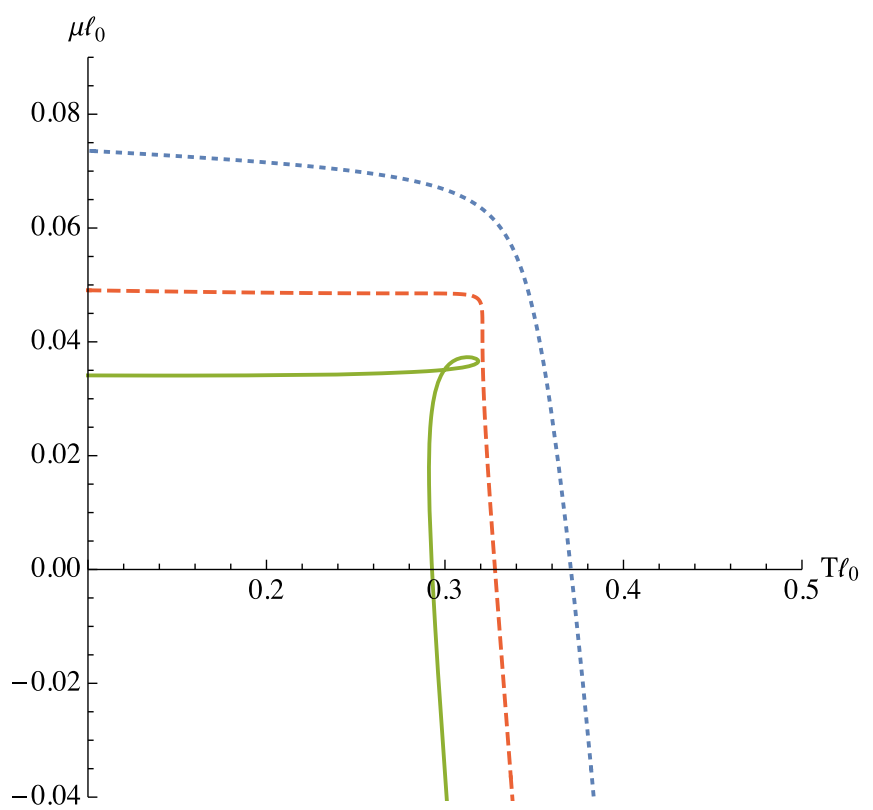

FIG. 1. $\mu-T$ diagram. The chemical potential is displayed as a function of $T \ell_{0}$ for fixed $P \ell_{0}^{4}=10$ and $C=20$ (blue dotted curve), $C=C_{c}=36$ (red dashed curve), and $C=60$ (green solid curve). Here, $Q=1, k=16 \pi$, and $\ell_{0}$ is an arbitrary reference length scale.

To investigate critical behavior, we study the free energy $F=F(T, P, Q, C)$ given by

$$
\begin{aligned}
F & =M-T S=\frac{3 G Q^{2} l^{2}+l^{2} r_{+}^{2}-r_{+}^{4}}{4 G r_{+} l^{2}} \\
& =\frac{2 \pi r_{+}}{3} \sqrt{\frac{6 P C}{k}}-\frac{2 \pi P r_{+}^{3}}{3}+\frac{3 Q^{2}}{4 r_{+}}
\end{aligned}
$$

where we have employed the relations Eqs. (1) and (10) and $r_{+}=r_{+}(T, P, Q, C)$ is implicitly given by the second relation, Eq. (22). Fixing $Q$, the corresponding $F=$ $F(T, P, Q, C)$ can be plotted parametrically, with dimensionful quantities displayed in units of some fiducial length $\ell_{0}$. What matters for criticality is, in contrast to standard black hole chemistry [17], the value of the central chargethere exists a critical central charge $C_{c}$ above which $\left(C>C_{c}\right)$ we observe swallowtail behavior (for any $P$ ), whereas for $C<C_{c}$ the free energy is smooth (for any $P$ ). In other words, there is a first order phase transition for theories with a large central charge (or many degrees of freedom), but no such phase transition for theories with a small central charge (or few degrees of freedom); we illustrate this in Fig. 2.

To find this critical central charge, we impose

$$
\frac{\partial T}{\partial r_{+}}=0=\frac{\partial^{2} T}{\partial r_{+}^{2}}
$$

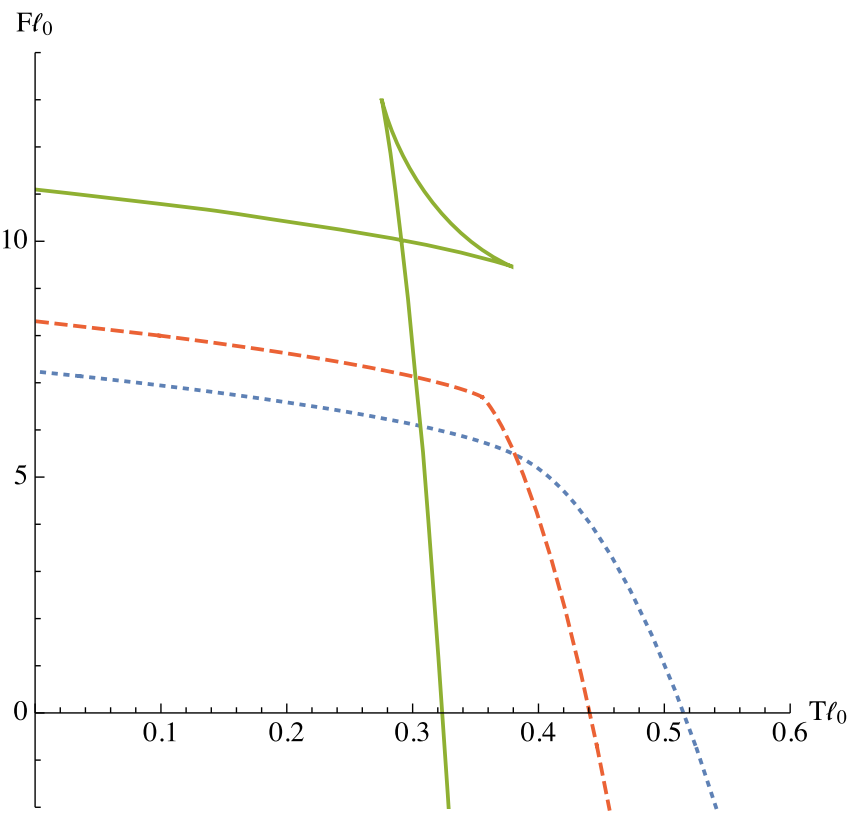

FIG. 2. Free energy diagram. $F-T$ diagram is displayed as a function of $C$ for fixed $P=15 \ell_{0}^{4}$. The dashed red corresponds to $C=C_{\text {crit }}=36$, the dotted blue is for $C=20$, and the solid green for $C=120$. Only theories with a large number of degrees of freedom admit a first order phase transition. The temperature of the critical point depends on $P$. We have set $Q=1$ and $k=16 \pi$.

corresponding to the "meeting" of the two cusps in the $F-T$ diagram. This yields

$$
C_{c}=\frac{9 k Q^{2}}{4 \pi}
$$

for the critical central charge, which is "universal"completely determined from $Q$ and independent of the thermodynamic pressure $P$.

In other words, charged AdS black holes demonstrate $\mu-C$ criticality with the corresponding equation of state

$$
C=C(\mu, T, P, Q)
$$

given implicitly by Eqs. (1), (10), and (22). In the vicinity of the critical point, the phase transition in the $\mu-C$ plane follows the corresponding Maxwell's equal area law, and the critical point itself is characterized by the mean field theory critical exponents [33].

While $C_{c}$ is universal, other characteristics of the critical point depend on the pressure. Namely, Eq. (27) yields

$$
r_{c}=\sqrt{6 G} Q, \quad l_{c}=6 \sqrt{G} Q, \quad T_{c}=\frac{\sqrt{6}}{18 \sqrt{G} \pi Q},
$$

which (apart from explicit factors of $G$ ) coincide with critical quantities obtained previously [7]. However, now 
we have a family of critical points as $G$ can be independently varied. Using, alternatively, Eq. (1), we obtain

$$
G=\frac{1}{\sqrt{96 \pi P} Q},
$$

and so we can vary $P$ to obtain this family. Other critical quantities can easily be expressed using Eqs. (30) and (31). In Fig. 3, we plot the coexistence curves in the $1 / C-T$ plane. We see that the corresponding critical points all share the same critical value of $C$, whereas the corresponding $T_{c}$ varies with pressure.

Summarizing, we have demonstrated that gauge-gravity duality provides a new understanding of the phase behavior of charged AdS black holes. We find that phase behavior is governed by a critical value of the central charge $C$ instead of the pressure. For a boundary theory with many degrees of freedom (a large central charge), there is a bulk first order phase transition, whereas for one with few degrees of freedom (a small central charge), there is not. The universality of the central charge (its independence of the bulk pressure) can be expected from dimensional analysis. It follows that the critical behavior of rotating black holes in any number of dimensions should also be universal. On the other hand, however, we may expect that for charged black holes in dimensions other than four, the critical central charge will depend on the bulk pressure.

We stress that there are important distinctions between the bulk $\mu-C$ criticality we have studied compared to

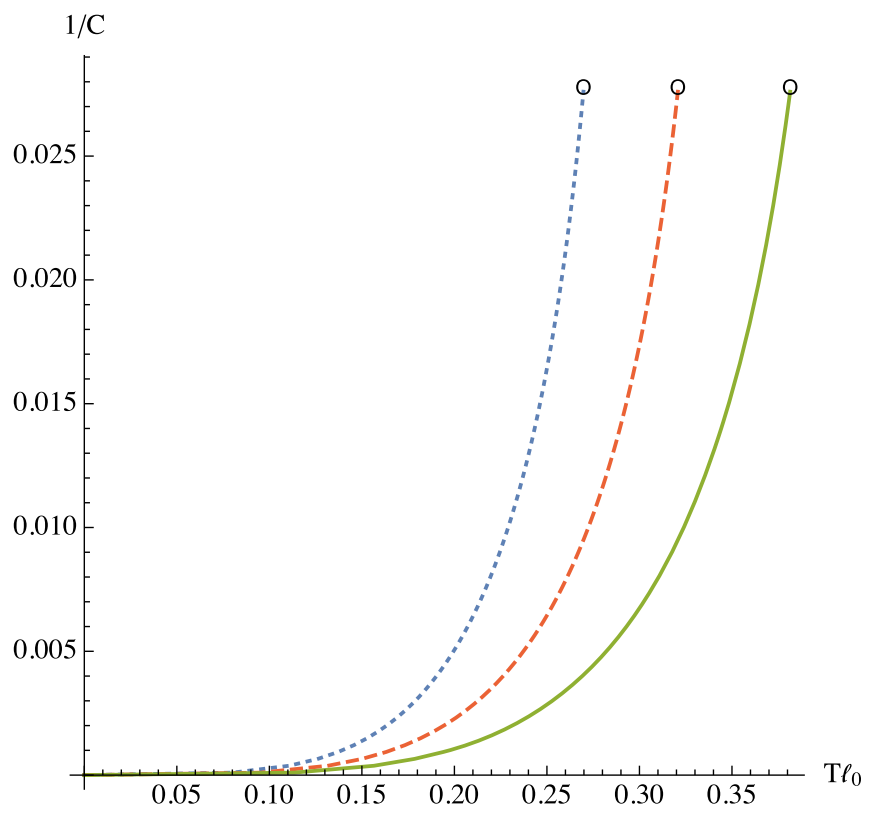

FIG. 3. Phase diagram. The coexistence curves in the $1 / C-T$ phase diagram are displayed for $P \ell_{0}^{4}=20$ (solid green), $P \ell_{0}^{4}=5$ (dotted blue), and $P \ell_{0}^{4}=10$ (dashed red), setting $Q=1$ throughout. These curves terminate at the corresponding critical points, which all share the same critical $C_{c}=36$ while the corresponding $T_{c}$ vary with pressure. previous investigations of holographic CFT [19,21,22,32], which employed the particular setting of $A d S_{5} \times S_{5}$ (or $A d S_{4} \times S_{7}$ ) and which derived $G$ from the fundamental (fixed) higher-dimensional theory by volume compactification. This leaves only one independent variation of $G$ and l. Furthermore, the CFT volume cannot vary (as the corresponding densities were used), and the CFT first law differs from Eq. (6). Our approach, based on Eq. (10), is more general: $G$ and $l$ vary independently.

We conclude with a number of open questions. For example, in this Letter we have concentrated on the mixed bulk first law, Eq. (18), where $P$ and $C$ were varied. What if instead one considered the variations of $G$ and $C$ ? In either setting, what happens to the more interesting phase transitions discovered in black hole chemistry such as reentrant phase transitions or isolated critical points? Are they still present? And if they are, are they governed by the universal critical central charge? What happens when the duality relation, Eq. (10), has to be generalized? Can we also incorporate the $1 / N$ corrections? These and many other questions remain interesting subjects for future investigation.

We would like to thank Manus Visser for helpful discussions on this work. This work was supported by the Perimeter Institute for Theoretical Physics and by the Natural Sciences and Engineering Research Council of Canada (NSERC). Research at the Perimeter Institute is supported in part by the Government of Canada through the Department of Innovation, Science and Economic Development Canada and by the Province of Ontario through the Ministry of Colleges and Universities.

* Corresponding author.

wcong@uwaterloo.ca

Corresponding author.

dkubiznak@perimeterinstitute.ca

Corresponding author.

rbmann@uwaterloo.ca

[1] J. M. Maldacena, The large N limit of superconformal field theories and supergravity, Adv. Theor. Math. Phys. 2, 231 (1998).

[2] S. W. Hawking and D. N. Page, Thermodynamics of black holes in anti-de Sitter space, Commun. Math. Phys. 87, 577 (1983).

[3] E. Witten, Anti-de Sitter space, thermal phase transition, and confinement in gauge theories, Adv. Theor. Math. Phys. 2, 505 (1998).

[4] A. Chamblin, R. Emparan, C. V. Johnson, and R. C. Myers, Charged AdS black holes and catastrophic holography, Phys. Rev. D 60, 064018 (1999).

[5] A. Chamblin, R. Emparan, C. V. Johnson, and R. C. Myers, Holography, thermodynamics and fluctuations of charged AdS black holes, Phys. Rev. D 60, 104026 (1999). 
[6] M. Cvetic and S. S. Gubser, Phases of R charged black holes, spinning branes and strongly coupled gauge theories, J. High Energy Phys. 04 (1999) 024.

[7] D. Kubiznak and R. B. Mann, P-V criticality of charged AdS black holes, J. High Energy Phys. 07 (2012) 033.

[8] D. Kastor, S. Ray, and J. Traschen, Enthalpy and the mechanics of AdS black holes, Classical Quantum Gravity 26, 195011 (2009).

[9] B. P. Dolan, The cosmological constant and the black hole equation of state, Classical Quantum Gravity 28, 125020 (2011).

[10] M. Cvetic, G. W. Gibbons, D. Kubiznak, and C. N. Pope, Black hole enthalpy and an entropy inequality for the thermodynamic volume, Phys. Rev. D 84, 024037 (2011).

[11] N. Altamirano, D. Kubiznak, and R. B. Mann, Reentrant phase transitions in rotating anti-de Sitter black holes, Phys. Rev. D 88, 101502(R) (2013).

[12] R. A. Hennigar, R. B. Mann, and E. Tjoa, Superfluid Black Holes, Phys. Rev. Lett. 118, 021301 (2017).

[13] B. P. Dolan, A. Kostouki, D. Kubiznak, and R. B. Mann, Isolated critical point from Lovelock gravity, Classical Quantum Gravity 31, 242001 (2014).

[14] N. Altamirano, D. Kubizňák, R. B. Mann, and Z. Sherkatghanad, Kerr-AdS analogue of triple point and solid/liquid/gas phase transition, Classical Quantum Gravity 31, 042001 (2014).

[15] S. W. Wei and Y. X. Liu, Triple points and phase diagrams in the extended phase space of charged Gauss-Bonnet black holes in AdS space, Phys. Rev. D 90, 044057 (2014).

[16] S.-W. Wei, Y.-X. Liu, and R. B. Mann, Repulsive Interactions and Universal Properties of Charged Anti-de Sitter Black Hole Microstructures, Phys. Rev. Lett. 123, 071103 (2019).

[17] D. Kubiznak, R. B. Mann, and M. Teo, Black hole chemistry: Thermodynamics with Lambda, Classical Quantum Gravity 34, 063001 (2017).

[18] C. V. Johnson, Holographic Heat Engines, Classical Quantum Gravity 31, 205002 (2014).

[19] B. P. Dolan, Bose condensation and branes, J. High Energy Phys. 10 (2014) 179.
[20] D. Kastor, S. Ray, and J. Traschen, Chemical potential in the first law for holographic entanglement entropy, J. High Energy Phys. 11 (2014) 120.

[21] J.-L. Zhang, R.-G. Cai, and H. Yu, Phase transition and thermodynamical geometry for Schwarzschild AdS black hole in $\mathrm{AdS}_{5} \times \mathrm{S}^{5}$ spacetime, J. High Energy Phys. 02 (2015) 143.

[22] J.-L. Zhang, R.-G. Cai, and H. Yu, Phase transition and thermodynamical geometry of Reissner-Nordström-AdS black holes in extended phase space, Phys. Rev. D 91, 044028 (2015).

[23] B. P. Dolan, Pressure and compressibility of conformal field theories from the AdS/CFT correspondence, Entropy 18, 169 (2016).

[24] F. McCarthy, D. Kubizňák, and R. B. Mann, Breakdown of the equal area law for holographic entanglement entropy, J. High Energy Phys. 11 (2017) 165.

[25] A. Karch and B. Robinson, Holographic black hole chemistry, J. High Energy Phys. 12 (2015) 073.

[26] M. Sinamuli and R. B. Mann, Higher order corrections to holographic black hole chemistry, Phys. Rev. D 96, 086008 (2017).

[27] M. R. Visser, Holographic thermodynamics requires a chemical potential for color, arXiv:2101.04145.

[28] D. Kastor, S. Ray, and J. Traschen, Smarr formula and an extended first law for lovelock gravity, Classical Quantum Gravity 27, 235014 (2010).

[29] One can think of this new form, which mixes the bulk and boundary (CFT) charges, as a choice of a new thermodynamic ensemble, with $C$ another bulk asymptotic charge.

[30] C. W. Misner, K. S. Thorne, and J. A. Wheeler, Gravitation (Macmillan, London, 1973).

[31] R. A. Hennigar, D. Kubizňák, and R. B. Mann, Entropy Inequality Violations from Ultraspinning Black Holes, Phys. Rev. Lett. 115, 031101 (2015).

[32] M. Chabab, H. El Moumni, and K. Masmar, On thermodynamics of charged AdS black holes in extended phases space via M2-branes background, Eur. Phys. J. C 76, 304 (2016).

[33] W. Cong, D. Kubizňák, and R. B. Mann, $\mu-C$ criticality of AdS black holes (to be published). 